\title{
Stimulus spacing, attention, and dimensional contrast
}

\author{
JOHN M. HINSON and CARI B. CANNON \\ Washington State University, Pullman, Washington.
}

\begin{abstract}
We examine some implications of an attentional model designed to explain dimensional contrast. Pigeons were trained to discriminate rectangular forms under conditions that produced positive dimensional contrast. In two experiments, the spacing of training stimuli was manipulated in ways that should have changed the allocation of attention. Experiment 1 compared the effect of an increased spacing between negative and positive stimuli with the effect of a decreased overall range of positive stimuli. Both manipulations increased the quality of discrimination performance. Experiment 2 demonstrated that dimensional contrast continued to occur under conditions that minimized the difficulty of the task. Results from these experiments were consistent with quantitative predictions of the attentional model concerning the interaction of discriminability factors and factors related to the allocation of attentional resources.
\end{abstract}

Most theories of stimulus control assume that performance in discrimination tasks is based on the underlying discriminability between stimuli (e.g., Balsam, 1988; D. S. Blough, 1983; Boneau \& Cole, 1967; Heinemann \& Chase, 1975; Pearce \& Redhead, 1993; Spence, 1937). Discriminability is determined by the way stimuli differ in value along specific physical dimensions or by the number and types of features that constitute different stimuli. It follows that discriminability increases as stimuli are increasingly separated along physical dimensions or as the number of shared features among the stimuli declines. One implication of this view of discriminability is what may be called the monotonicity assumption. The monotonicity assumption holds that there is a monotonic relation between measures of discriminability and measures of performance in discrimination tasks. As discriminability between stimuli increases, actual discrimination performance should also improve.

Although the monotonicity assumption is a reasonable starting point, many findings in the conditioning literature are inconsistent with this assumption. One important violation of the monotonicity assumption appears in the phenomenon of dimensional contrast. Dimensional contrast refers to an enhancement of discrimination performance for relatively similar positive $(\mathrm{S}+)$ and negative $(\mathrm{S}-)$ training stimuli, as compared with more dissimilar stimuli. For example, D. S. Blough (1975) found that pigeons trained on a maintained generalization procedure with multiple wavelength stimuli showed two related effects: positive and negative dimensional contrast. Positive dimensional contrast refers to the finding that the highest responding occurred during $\mathrm{S}+$ stimuli near $\mathrm{S}-$ on the wavelength

Correspondence concerning this article should be addressed to J. M. Hinson, Department of Psychology, Washington State University, Pullman, WA 99164-4820 (e-mail: hinson@mail.wsu.edu) continuum. Negative dimensional contrast refers to the finding that the lowest responding was found for S - stimuli near $\mathrm{S}+$ along the wavelength continuum.

Dimensional contrast is regularly observed during assessment of dimensional.stimulus control with maintained generalization procedures (e.g., D. S. Blough, 1975, 1983; Catania \& Gill, 1964; Farthing, 1974; Hinson \& Higa, 1989a; Hinson \& Malone, 1980; Hinson \& Tennison, 1997; Reynolds, 1961). The effect has been reported during training with many different stimulus continua, including angular orientation, spatial position, wavelength of visible light, and visual flicker-rate (e.g., D. S. Blough, 1975; Catania \& Gill, 1964; Farthing, 1974; Hinson, 1988; Hinson \& Higa, 1989a; Reynolds, 1961). There is no obvious dependence of the effect on unusual reinforcement contingencies or on peculiarities of the training procedure. Also, dimensional contrast is typically a large effect. In work on positive dimensional contrast with wavelength stimuli, we have routinely obtained effects in the range of $50 \%-100 \%$ increases in response rate (e.g., Hinson \& Tennison, 1997). That is, an $S+$ quite similar to $S-$ routinely maintained about twice the response rate as other $\mathrm{S}+$ stimuli quite dissimilar from $\mathrm{S}-$. This enhancement occurred despite the fact that all $\mathrm{S}+$ values provided exactly the same scheduled and obtained rates of reinforcement for responding.

The first serious theoretical account of dimensional contrast was provided by D. S. Blough (1975, 1983). Blough's incremental model uses a linear operator learning mechanism derived from the theory of conditioning proposed by Rescorla and Wagner (1972). The model predicts dimensional contrast on the basis of a generalization parameter that describes interactions among stimuli along a continuum. Similar stimuli are presumed to share response strength, and it is the dynamics of this sharing process that accounts for dimensional contrast. According to the incremental model, if $\mathrm{S}-$ and $\mathrm{S}+$ are widely spaced along 
a stimulus continuum, making them very dissimilar, there should be limited sharing of strength between $\mathrm{S}-$ and $\mathrm{S}+$. As a result, the model correctly predicts a decrease in the size of dimensional contrast as $\mathrm{S}-$ and $\mathrm{S}+$ are more widely separated along the continuum (P. M. Blough, 1980; Essock \& Blough, 1977).

Blough's incremental model does a reasonably good job of accounting for the general features of dimensional contrast (e.g., D. S. Blough, 1975). Nonetheless, from its inception, it has been recognized that the model usually predicts a smaller magnitude of dimensional contrast than is actually observed (e.g., D. S. Blough, 1983). One implication is that the incremental model may be omitting additional factors that contribute to dimensional contrast.

We have previously suggested that attention plays an important role in dimensional contrast (Hinson \& Tennison, 1997). In our view, the enhancement of discrimination between an $\mathrm{S}-$ and an $\mathrm{S}+$ that are relatively similar is a reflection of increased attention to these stimuli. This enhancement of performance can be viewed as either an increased allocation of processing resources (e.g., Hirst \& Kalmar, 1987; Navon, 1984) or an increased focus of an attentional aperture (e.g., Briand \& Klein, 1987; Posner, Snyder, \& Davidson, 1980). In either case, we assume that there is some limit on total attention, so that greater attention to some stimuli means less attention available for other stimuli.

We have found it most useful to conceive of attention as a gradient that extends over an internal representation of stimuli presented during training. This notion of attention is conceptually related to mechanisms invoked in the human visual search literature, involving the operation of attentional "spotlights" or gradients within the search field (e.g., LaBerge \& Brown, 1989). Attention so conceived is also reminiscent of the kinds of limitations discussed by Heinemann and his colleagues in their models of discrimination and pattern recognition (e.g., Heine- mann \& Avin, 1973; Heinemann, Avin, Sullivan, \& Chase, 1969; Heinemann \& Chase, 1990).

Figure 1 illustrates how a gradient of attention could produce dimensional contrast during a task in which one stimulus is being discriminated from other stimuli along an ordered continuum. The left panel of Figure 1 shows the ordered stimulus continuum, along with two hypothetical functions. A solid line, labeled the attention function, shows high attention in some local regions, such as near $\mathrm{S}-$, and lower attention for stimuli more widely displaced from $\mathrm{S}-$. A dashed line, labeled the discriminability function, represents the relative ease of distinguishing $S-$ from the other $\mathrm{S}+$ stimuli. Stimuli farther from $\mathrm{S}-$ along the continuum are more discriminable from it, so the function increases with distance away from $\mathrm{S}-$.

Actual discrimination performance depends on the joint impact of the attention function and the discriminability function. In other words, the quality of performance will be based on how easy it is to distinguish one stimulus from another and how much attention is applied to the task. These joint contributions are illustrated in the right panel of Figure 1. A solid line represents the product of the values of the attention function and the discriminability function at each point along the stimulus continuum. The resulting performance function is nonmonotonic, with the best performance for $\mathrm{S}+$ near $\mathrm{S}-$, and poorer performance with $\mathrm{S}+$ values farther along the continuum. Thus, the preferential allocation of attention improves overall performance in areas of the continuum where discrimination is relatively difficult.

We have formalized these general notions to make more specific predictions (see Hinson \& Tennison, 1997). In brief, the model defines the discriminability function as

$$
d=1-e^{(-x * s)} \text {, }
$$

where $d$ is the potential level of discrimination between $\mathrm{S}-$ and $\mathrm{S}+, s$ is the distance between $\mathrm{S}-$ and $\mathrm{S}+$ along
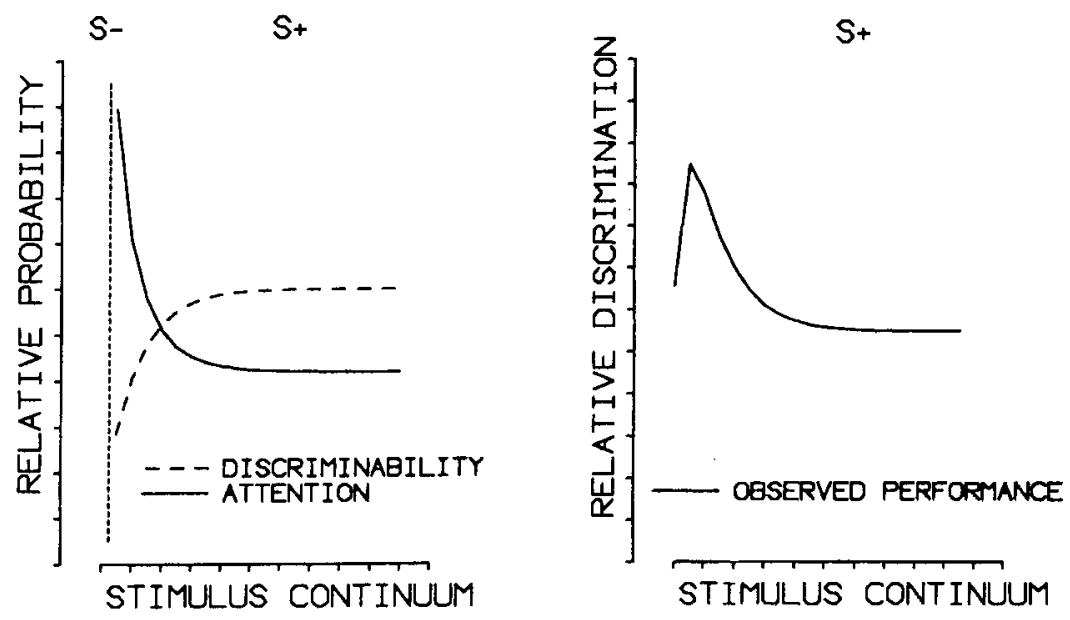

Figure 1. Graphic representation of the attentional model of dimensional contrast. Left panel: hypothetical discriminability and attention functions. Right panel: resulting discrimination performance. 
a physical dimension, $x$ is a growth parameter, and $e$ is the base of natural logarithms.

The attention function is given as:

$$
a=e^{[y *(1-d)]} * z
$$

where $y$ is a decay parameter for the decline of attention and $z$ is a parameter that reflects the asymptotic level of attention. The exponential term $(1-d)$, derived from Equation 1, provides the distance over which attention is dispersed. Actual measures of discrimination are reflected in the performance function, given by

$$
p=a * d
$$

It is worth considering how each parameter of the model is defined and how each contributes to performance. Equation 1 states that discriminability between $\mathrm{S}-$ and $\mathrm{S}+$ grows as $\mathrm{S}-$ and $\mathrm{S}+$ are more widely separated along a given physical dimension. The greater the difference between $\mathrm{S}-$ and a given $\mathrm{S}+$, the larger the value of $s$ and, therefore, the higher the value of $d$. The parameter $x$ is a scaling factor that reflects the specific units of measure of the physical dimension. We assume that the value of $x$ should remain the same, despite changes in other variables, such as reinforcement frequency, schedules, or session duration. Equation 1 is a simplified conception based on earlier findings that an exponential function provided a good description of stimulus dissimilarities in work on stimulus generalization (e.g., Shepard, 1987). Ultimately, Equation 1 has been justified by its usefulness in accounting for actual results (e.g., Hinson, Cannon, \& Tennison, 1999; Hinson \& Tennison, 1997).

Equation 2 states that attention is a decay function. The breadth of the function is determined by the value of $(1-d)$ taken from Equation 1. That is, the greater the difference between $\mathrm{S}-$ and $\mathrm{S}+$ along the stimulus continuum, the more widely attention is distributed. In addition, Equation 2 indicates that attention is greatest where the physical difference between $\mathrm{S}-$ and $\mathrm{S}+$ is smallest and that attention declines as $\mathrm{S}+$ becomes increasingly different from $\mathrm{S}-$ along the physical dimension. By convention, we refer to the dimensional border as the region of the stimulus continuum between $\mathrm{S}-$ and $\mathrm{S}+$ where the attention function peaks. The more $\mathrm{S}+$ is separated from the border, the more the attention function will have declined. Finally, the specific values of parameters $y$ and $z$ should be determined by the attentional resources available to the individual.

It is important to note that Equations 1 and 2 are functional models. Equation 1 describes the result of an unspecified set of mechanisms that contribute to discriminability. Equation 2 describes the action of what are, presumably, attentional mechanisms. On the basis of ordinary notions of attention mentioned earlier, we assume that attentional resources are limited. If this assumption is correct, the area under the attention gradient should remain constant for a fixed level of available attention. A fixed level of total attention would be reflected by an in- verse relation between the two parameters of Equation 2. In all of our prior work, the two parameters of Equation 2 have been inversely related (Hinson et al., 1999; Hinson \& Tennison, 1997).

Although our attentional account is consistent with the finding of dimensional contrast, we must also demonstrate that the model adds to, or improves upon, prior models. One way to evaluate the attentional model is to examine predictions of this model that are not expected from other theories. One such distinct prediction concerns stimulus spacing factors. Because it is a spatial model, the attentional model suggests that two aspects of stimulus spacing are important in determining the allocation of attention and, thereby, dimensional contrast. One aspect is the spacing between $\mathrm{S}-$ and $\mathrm{S}+$ along the dimensional border, which we refer to as the criterial range. A second aspect is the overall range of variation of $\mathrm{S}+$ stimuli, which we refer to as the stimulus range.

Discrimination performance will ordinarily improve with increases in criterial range. An increase in the difference between $\mathrm{S}$ - and $\mathrm{S}+$ should make these stimuli easier to distinguish. We also argue that when criterial range increases, the dimensional border changes. The attention function then shifts to the new border. As a result, performance should improve, owing to the joint effects of changes in the allocation of attentional resources and the greater ease of distinguishing $\mathrm{S}-$ from $\mathrm{S}+$. But note that, with very large increases in criterial range, performance can decline, owing to the very wide dispersal of attentional resources (see, also, Tennison \& Hinson, 1993).

The attentional model assumes limited attentional resources, and it assumes that these resources are distributed along a spatial gradient. As a consequence, if stimulus range is relatively large, limited resources must be allocated over a wider domain at a lower level. On the other hand, if stimulus range is small, attentional resources can be allocated over a narrower domain at a higher level. Blough's incremental model provides only a limited role for stimulus factors, in that the positive and the negative stimuli must be reasonably similar in order for dimensional contrast to occur. The incremental model predicts that, as criterial range increases, the magnitude of dimensional contrast should decline. Overall stimulus range is predicted to have no independent effect (see, e.g., D. S. Blough, 1983; P. M. Blough, 1980).

The attentional model expects an impact of stimulus spacing factors that is not implied by Blough's incremental model. The attentional model predicts an interaction between the effects of the criterial and the stimulus range. Dimensional contrast, by this account, depends on the joint impact of both types of stimulus spacing (Hinson \& Tennison, 1997).

The present studies assess whether the interactions predicted by the attentional model occur and whether these effects are consistent with quantitative features of the model. All the experiments reported here are based on discriminations of visual forms. These stimuli were selected to ex- 
tend previous findings to a new stimulus continuum. In Experiment 1, we examine the impact of increases in criterial range and of decreases in stimulus range. In Experiment 2 , we further examine stimulus spacing effects in a situation in which the impact of other training factors is minimized.

\section{EXPERIMENT 1}

\section{Method}

Subjects. Twelve homing pigeons with varied training histories served as subjects. The birds were maintained at $80 \%$ of their freefeeding weights.

Apparatus. The experiments were carried out in a Campden Instruments, three-key operant conditioning chamber with internal dimensions of $35 \times 35 \times 33 \mathrm{~cm}$. The center key was located $27.5 \mathrm{~cm}$ above the floor and $16 \mathrm{~cm}$ from either side of the chamber. It was made of clear Plexiglas and provided a circular viewing region with a diameter of approximately $4 \mathrm{~cm}$. Black tape covered the two side keys. A 6-W houselight provided diffuse illumination throughout the chamber during experimental sessions. A $5 \times 6 \mathrm{~cm}$ aperture, located in the middle of the front panel $13.5 \mathrm{~cm}$ from the floor, directly below the center key, provided access to a food magazine. Mixed grain presented for $3 \mathrm{sec}$ was used as a reinforcer. The experimental chamber was part of a larger sound-proofing box, which contained a fan for ventilation and for masking of extraneous noise.

An IBM XT-type computer, along with a dedicated, single-board microcomputer, controlled real-time contingencies during the experiment and collected all the data. The stimuli were displayed on an attached monochrome monitor. The $14-$ in. monitor had a dot pitch of $0.39 \mathrm{~mm}$, with a resolution of $640 \times 480$ pixels at a $60-\mathrm{Hz}$ refresh rate. All the stimuli were drawn from lines 1 pixel in width, appearing in a yellow phosphor. A double-lens assembly projected the screen image to the response key. Inspection of the stimuli by the experimenters indicated that the projected form appeared in a totally black background. To the human eye, the form looked to be slightly behind the response key, although there were no other strong indications of depth. Movement of viewing position several inches vertically and horizontally had no obvious impact on the quality of the stimulus as it appeared on the key. Size measurements of the stimuli were made on the front of the key.

Procedure. After preliminary autoshaping in the chamber, to develop some responding to the key, training for Experiment 1 began. Stimulus 1 was designated $S-$, whereas Stimuli 2-13 were designated $\mathrm{S}+$. Responses during $\mathrm{S}-$ and $\mathrm{S}+$ were reinforced according to a probabilistic fixed-interval (FI) 20 -sec schedule. For $S+$, the first response occurring at least $20 \mathrm{sec}$ after the onset of the visual stimulus always resulted in a 3 -sec access to mixed grain. For $\mathrm{S}-$, the first response occurring at least $20 \mathrm{sec}$ after the onset of the visual stimulus produced food reinforcement on $3 \%$ of the presentations. On the other $97 \%$ of the presentations of S - , a 3-sec interval with houselight off was provided, instead of a 3 -sec access to food. Note that the FI contingency employed in this task required that each stimulus presentation be ended with a response. Therefore, a low probability of reinforcement, rather than zero probability, was used during $S-$ trials, to ensure some consistent responding during $S-$.

The 12 birds were divided into three groups of 4 each. All the birds were trained to discriminate $S-$ from $S+$ on the basis of width of the rectangular form. Sets of training stimuli for different groups of birds had the same numbers of stimuli but different spacing along the width continuum. The stimuli for each group are schematically represented in Figure 2. The stimuli for Group 1, labeled baseline, provided a relatively small criterial range (i.e., a narrow border between $\mathrm{S}-$ and $\mathrm{S}+$ ) and a relatively large stimulus range (i.e., a wide spacing of $\mathrm{S}+$ along the stimulus dimension). For Group 2, labeled

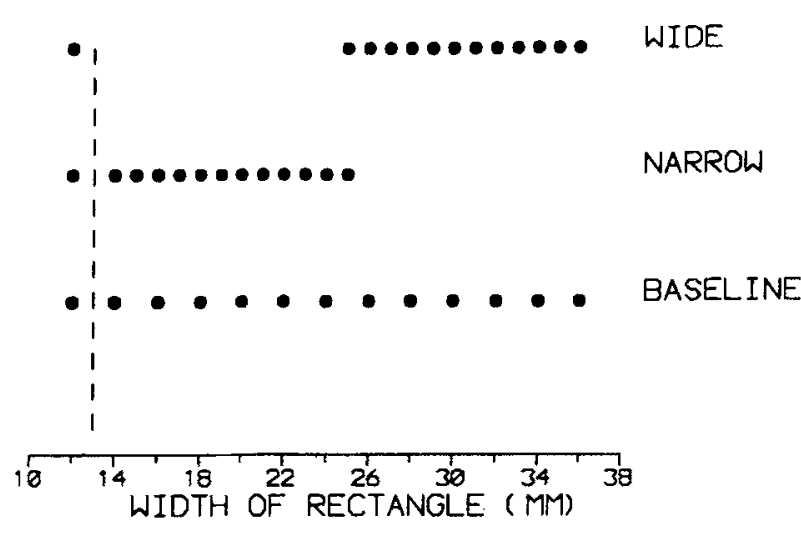

Figure 2. Schematic representation of the rectangular stimuli used for the three training groups of Experiment 1 .

narrow, training stimuli had the same criterial range as the baseline group but a relatively narrower stimulus range. For Group 3, labeled wide, training stimuli had a relatively wide criterial range but the same stimulus range as the narrow group. The height of every visual form was $10 \mathrm{~mm}$.

There were 120 stimulus presentations during each session. The sequence of the stimuli was determined by a pseudo-random process, with a .5 probability of selecting either $\mathrm{S}-$ or $\mathrm{S}+$. Each bird received 14 daily sessions conducted 6 or 7 days a week. Responding appeared stable after 7 sessions, as is evidenced by the lack of a trend in responding from session to session and by small changes in absolute responding from session to session-that is, less than $5 \%$ change from one session to the next. The stability criteria are consistent with those used in our prior studies of visual wavelength discrimination (e.g., Hinson \& Tennison, 1997).

\section{Results and Discussion}

The results from Experiment 1 are presented in the form of relative discrimination ratios, rather than in absolute response rates. We prefer a relative measure of performance because absolute response rates can fluctuate, owing to a variety of motivational factors (e.g., satiation) and because previous work suggests that relative discrimination functions are a more consistent measure of dimensional contrast (e.g., Hinson, 1988). Moreover, the attentional model is more concerned with the relative accuracy of discrimination, rather than with how much responding occurs.

Figure 3 provides mean discrimination functions for each group of birds, averaged over the last seven sessions of training. To produce the discrimination functions, mean response rates were obtained by dividing the total number of responses during each stimulus by the total number of presentations of that stimulus. The mean response rate for each $\mathrm{S}+$ was then converted into a discrimination ratio, using the formula

$$
P_{\mathrm{S}^{+}}(n)=r_{\mathrm{S}^{+}}(n) /\left(r_{\mathrm{S}^{+}}(n)+r_{\mathrm{S}_{-}}\right),
$$

where $r_{\mathrm{S}+}(n)$ is response rate during each of the $(n) \mathrm{S}+$ stimuli, and $r_{\mathrm{S}-}$ is response rate during the $\mathrm{S}-$ stimulus. Thus, the ratio is a proportion where .5 indicates equal re- 


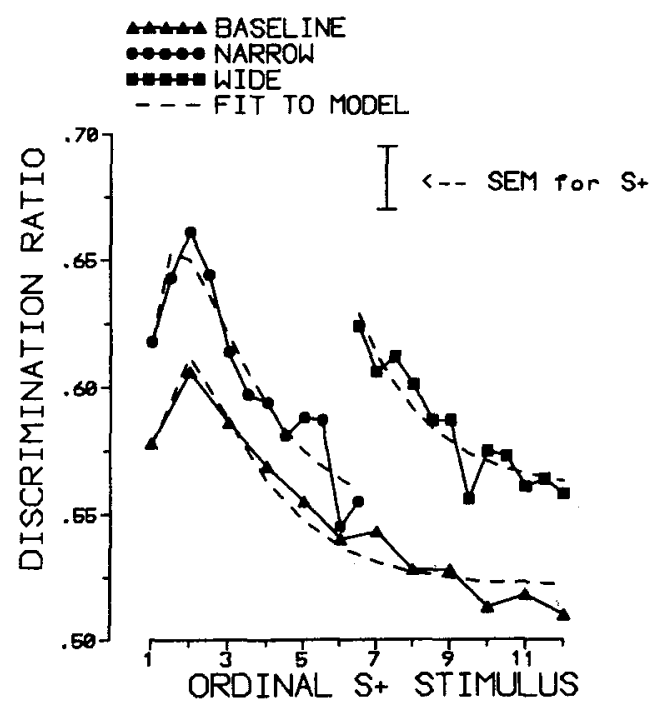

Figure 3. Group results from Experiment 1. Solid lines: actual discrimination performance for each training group. Dashed lines: best fits of the attentional model based on Equation 7. The vertical bar represents plus and minus one standard error of the mean for discrimination ratios.

sponding during $\mathrm{S}-$ and a given $\mathrm{S}+$, and values greater than .5 indicate increasingly greater responding during $\mathrm{S}+$, relative to $\mathrm{S}-$.

For convenience in fitting the model to the results, we standardized the values of rectangular width for each $\mathrm{S}+$. Parameter $s$ of Equation 1 reflects the difference between $\mathrm{S}-$ and $\mathrm{S}+$ along the rectangular width continuum. We defined the value of $s=1$ as a difference of $2 \mathrm{~mm}$ in rectangular width between $\mathrm{S}-$ and $\mathrm{S}+, s=2$ as a 4-mm difference, $s=3$ as a 6-mm difference, and so on. In the baseline condition, $\mathrm{S}-$ was $12 \mathrm{~mm}$ in width, whereas the 12 $\mathrm{S}+$ values in the baseline condition varied from 14 to $36 \mathrm{~mm}$ in width. Therefore, the $12 \mathrm{~S}+$ stimuli were given $s$ values of $1-12$, to reflect their width differences from $\mathrm{S}-$. Accordingly, the value of $s$ for $\mathrm{S}+$ stimuli in the narrow condition varied from 1 to 6.5 , and the value of $s$ in the wide condition varied from 6.5 to 12 . These standardized values appear on the abscissa of Figure 3.

The discrimination functions for each group show positive dimensional contrast. Discrimination is best for $\mathrm{S}+$ near $\mathrm{S}-$, rather than for $\mathrm{S}+$ farthest away from $\mathrm{S}-$. Compared with the baseline group, overall performance is better in the narrow group and in the wide group. That is, discrimination improved with either a decrease in stimulus range or an increase in criterial range, apart from any other factor. These overall effects are consistent with previously described assumptions of the attentional model (e.g., Hinson \& Tennison, 1997).

The attentional model was fit to results in Figure 3, using the Quasi-Newton, nonlinear estimation procedure of SYSTAT statistical software (SYSTAT, Evanston, IL).
The Quasi-Newton method minimizes a least-squares loss function by means of a numeric estimate of derivatives of the function. The discrimination function for each group was fit to Equation 3, which combines the three free parameters of Equations 1 and 2.

To assess the statistical reliability of differences in parameters between conditions, we compared $95 \%$ confidence intervals. The nonlinear estimation procedure in SYSTAT provides asymptotic standard errors for each parameter. Confidence intervals were calculated from the obtained standard errors and the degrees of freedomnamely, one less than the number of stimuli in the S+ response function (see, e.g., Hays, 1988). For a difference in parameters to be considered reliable by this measure, there could be no overlap in the confidence intervals for each estimated parameter. Obtained parameter values and quality of fit appear in Table 1.

Table 1 shows that Equation 3 provides good fits to the results from the baseline and narrow conditions, evidenced by high corrected $r^{2}$ values. In addition, the value of parameter $x$ from the discriminability function in Equation 1 is the same for these two groups. This finding is consistent with our assumption that the same discriminability function should describe training with a common set of stimuli. The only anticipated change between the baseline and the narrow conditions is a change in the relative allocation of attentional resources, owing to the smaller stimulus range for the narrow group. This expectation is consistent with the obtained parameter values. The higher discrimination level of the narrow group, as compared with the baseline group, is accounted for by an increase in parameter $z$ of Equation 2. Parameter $z$ represents a general multiplier to the attentional function, with higher values representing greater attentional resources. Therefore, the improvement in performance for the narrow group can be attributed to the restricted dispersal of attentional resources.

Equation 3 did not provide an acceptable fit to the results from the wide group, in that there was no convergence to a solution with nonzero variance accounted for. This outcome is not surprising. The original model was not designed to accommodate changes in criterial range. In fact, for simplicity, the model assumes that the distance between $\mathrm{S}-$ and $\mathrm{S}+$ is about the same as the distance be-

Table 1

Estimate of Parameter Values, Corrected Variance Accounted For, and Asymptotic Standard Errors, for Fits to Equation 3

\begin{tabular}{|c|c|c|c|c|c|c|c|}
\hline \multirow[b]{3}{*}{ Condition } & \multicolumn{6}{|c|}{ Parameter Value } & \multirow[b]{3}{*}{ Fit $\left(r^{2}\right)$} \\
\hline & \multicolumn{2}{|c|}{$x$} & \multicolumn{2}{|c|}{$y$} & \multicolumn{2}{|c|}{$z$} & \\
\hline & Est & $S E$ & Est & $S E$ & Est & $S E$ & \\
\hline Baseline & $.50^{*}$ & .03 & $1.70^{*}$ & .03 & $.52^{*}$ & .004 & .95 \\
\hline Narrow & $.50^{*}$ & .05 & $1.74^{*}$ & .06 & $.55+$ & .011 & .91 \\
\hline Wide & 2.09 & - & 1.08 & - & .58 & - & .00 \\
\hline
\end{tabular}

Note-Different superscripts indicate parameter values that differ from one another outside a $95 \%$ confidence interval. 
tween $\mathrm{S}+$ and the peak of the attention function. That is why the distance term, $(1-d)$, derived from Equation 1, appears in Equation 2.

A simple way to accommodate movement of the peak of the attention function is to add a parameter that reflects this movement. To begin with, Equation 1 represents the distance between $\mathrm{S}-$ and $\mathrm{S}+$. If the peak of the attention function moves away from $\mathrm{S}-$ as the criterial range increases, the distance between $S+$ and the peak must be smaller than the distance between $\mathrm{S}-$ and $\mathrm{S}+$. Hence, we can modify Equation $I$ to

$$
d p=1-e^{[-x *(s-c)]},
$$

where $d p$ is the distance between the peak of the attention function and the $\mathrm{S}+$ border and $c$ represents the displacement of the peak within the criterial range. The new form of Equation 2 becomes

$$
a^{\prime}=e^{[v *(1-d p)]} * z,
$$

and the revised performance function is

$$
p=a^{\prime} * d,
$$

where $a^{\prime}$ reflects the modified version of the attention function.

As was noted earlier, when the criterial range increases, the dimensional border between $\mathrm{S}-$ and $\mathrm{S}+$ changes. We presume that the peak of the attention function will move to accommodate the new border. In practical terms, parameter $c$ in Equation 5 represents how far the attention function has moved with the increase in the criterial range. That is, as $c$ increases above zero, the distance between the peak of the attention function and $\mathrm{S}+$ will be smaller than the distance between $\mathrm{S}+$ and $\mathrm{S}-$ at the dimensional border. Hence, the level of attention near $\mathrm{S}+$ will be relatively higher than it would be if the peak of the attention function had not moved. So, for example, if the criterial range increases to 5 units, the value of $c$ should be 2.5 units to move the attention function exactly to the new dimensional border.

The new parameter $c$ in Equation 5 makes a total of four free parameters, which is obviously too many for a sensible fit. However, we can constrain the fit on the basis of our previous assumptions about how attention is allocated. Parameter $z$ can be set to .5 on the basis of the results from

Table 2

Estimate of Parameter Values, Corrected Variance Accounted For, and Asymptotic Standard Errors, for Fits to Equation 7

\begin{tabular}{lllllll}
\hline & \multicolumn{4}{c}{ Parameter Value } & \\
\cline { 2 - 3 } Condition & \multicolumn{2}{c}{$z$} & & \multicolumn{2}{c}{$c$} & \\
\cline { 2 - 3 } & Est & $S E$ & & Est & $S E$ & Fit $\left(r^{2}\right)$ \\
\hline Baseline & $.52^{*}$ & .003 & & $.001^{*}$ & .03 & .95 \\
Narrow range & $.55 \dagger$ & .005 & & $.051^{*}$ & .04 & .91 \\
Wide border & $.56 \dagger$ & .004 & & $1.74 \dagger$ & .19 & .86 \\
\hline
\end{tabular}

Note-Different superscripts indicate parameter values that differ from one another outside a $95 \%$ confidence interval. Parameter $x$ was set at .5 , and parameter $y$ was set at 1.7 , to constrain the fit the baseline group and our assumption of an invariant discriminability function. Also, because parameter $y$ did not change between the baseline and the narrow groups, we can establish its value at 1.7 . We are left with two free parameters: $z$, representing overall changes in the level of attentional resources, and $c$, reflecting the displacement of the peak of the attention function.

Results of the two-parameter fit of Equation 7 appear in Table 2. Estimates of the best-fitting functions are plotted as dashed lines in Figure 3, along with the obtained discrimination functions for each group. There are no changes in the quality of fit to the baseline and the narrow groups, but the wide group now shows a reasonably high $r^{2}$ value. The value of parameter $z$ for the wide group is different from that for the baseline group, but not reliably different from that for the narrow group. Thus, with a comparable stimulus range, the value of $z$ is equivalent in the wide and the narrow groups.

Parameter $c$ does not differ reliably between the baseline and the narrow groups, and in neither case is the parameter reliably different from zero. But $c$ is reliably larger in the wide group, as compared with the baseline or the narrow group. If the peak of the attention function had moved to the exact center of the criterial range, we would expect $c$ to increase by 2.75 ordinal stimulus units-in other words, half of the distance between $\mathrm{S}-$ and $\mathrm{S}+$. The obtained value of $c$ that provided the best fit of Equation 7 for the wide group was 1.74 , indicating that the change in $c$ is in the predicted direction but lesser in magnitude than would be expected from exact centering of the function. Nonetheless, the general point remains that the peak of the attention function does appear to move when criterial range increases.

\section{EXPERIMENT 2}

Like prior studies of dimensional contrast, Experiment 1 employed a fairly difficult discrimination task, using large numbers of closely spaced stimuli during each session. One may reasonably wonder whether dimensional contrast occurs only in the most challenging discrimination settings. To the contrary, the attentional model argues that dimensional contrast is not due to some absolute level of difficulty in discriminating $S-$ from $\mathrm{S}+$ but, rather, to the interaction of discriminability with the attentional resources that are available. If the attentional resources are limited, dimensional contrast may be found even in relatively easy tasks.

Experiment 2 employs a procedure that reduces some of the typical difficulties of discrimination training. In this experiment, only two stimuli were used during each session. Also, the training regimen was changed, to enhance the benefit of carry-over across each training session. These aspects of the experiment should contribute to higher discrimination among stimuli. At the same time, Experiment 2 employs relatively wide spacing of training stimuli. As in Experiment 1, wide spacing of stimuli should reduce the attentional resources available for the discrim- 


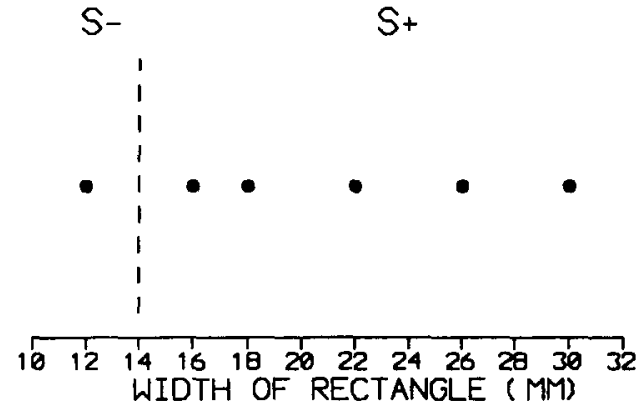

Figure 4. Schematic representation of the rectangular stimuli used during Experiment 2.

ination task and should contribute to the production of dimensional contrast.

\section{Method}

Subjects. Five homing pigeons with varied training histories served as subjects. The birds were maintained at $80 \%$ of their freefeeding weights.

Apparatus. The experiments were carried out in the apparatus described for Experiment 1.

Procedure. The experiment began without any special preliminary training. During each experimental session, a pair of training stimuli was presented. Stimulus 1 was designated S-, whereas Stimulus 2 was designated $\mathbf{S}+$. Responses during $\mathrm{S}-$ and $\mathrm{S}+$ were reinforced according to the contingencies described for Experiment 1 .

During each session, Stimulus $1(\mathrm{~S}-)$ was the same rectangular form. The value of Stimulus $2(\mathrm{~S}+)$ was one of five different rectangles that varied from $\mathrm{S}-$ along the dimension of width. The height of every visual form was $10 \mathrm{~mm}$. These stimuli are schematically illustrated in Figure 4.

Although constant for a given session, the value of $\mathrm{S}+$ changed across successive sessions. That is, during each consecutive session, $\mathrm{S}+$ was made increasingly different from $\mathrm{S}-$. After the session with the $S+$ that was most different from $S-$, the cycle was reversed, and the $\mathrm{S}+$ stimuli that appeared in succeeding sessions were progressively more similar to $\mathrm{S}-$. The cycle was continued throughout the 54 sessions of training in Experiment 2. This procedure was intended to make the task within each session as easy as possible, while still having the birds discriminate a reasonably wide range of stimuli across all the sessions.

There were 120 stimulus presentations during each session. The sequence of stimuli was determined by a pseudo-random process, with a .5 probability of selecting either $\mathrm{S}-$ or $\mathrm{S}+$. Sessions were conducted 5 or 6 days a week. Using the criteria described in Experiment 1 , responding appeared stable, with no obvious systematic changes, after roughly 1 week of training.

\section{Results and Discussion}

The results from Experiment 2 have been collapsed across birds for the final seven sessions of training with each $\mathrm{S}+$. As before, a discrimination ratio was calculated on the basis of Equation 4. Discrimination performance functions appear in Figure 5 with a solid line and solid symbols. Note that the standardized units for rectangular width that were used in Experiment 1 are also used for each $\mathbf{S}+$ in Experiment 2. The clearest result in Figure 5 is the bitonic discrimination function indicative of posi- tive dimensional contrast. Best discrimination occurs with an intermediate value of $\mathrm{S}+$ along the rectangular width continuum.

Because of the restricted range of $\mathrm{S}+$ during each session of Experiment 2, we expected that discrimination ratios should be higher than those in any of the conditions of Experiment 1. A comparison of the results in Figure 5 with those in Figure 3 shows that performance for each pair of stimuli is better in Experiment 2.

To assess the fit of the attentional model to each pair of $\mathrm{S}-$ and $\mathrm{S}+$ stimuli, we used the following procedure. Because Experiments 1 and 2 use the same type of rectangular forms, the value of the discriminability parameter $x$ should be the same for the two experiments. Therefore, we used the value of .5 for $x$, obtained in Experiment 1, as a constant.

Next, in Experiment 2 the dispersal of attention is limited by using only two stimuli during each session. We estimated values for the attentional parameters, $y$ and $z$ of Equation 2, for the $S-$ and $S+$ stimuli closest to one another along the continuum. According to the model, this should represent the highest level of attention. The estimated parameter values are $y=.74$ and $z=.69$. From these values, we extrapolated the level of attentional dispersal for the other S+ stimuli use in Experiment 2.

Two theoretical functions appear in Figure 5, along with the function for actual performance. The coarse dashed line is the prediction when the peak of the attention function is centered exactly between the $\mathrm{S}-$ and $\mathrm{S}+$, at the dimensional border, during each session. The fine dashed

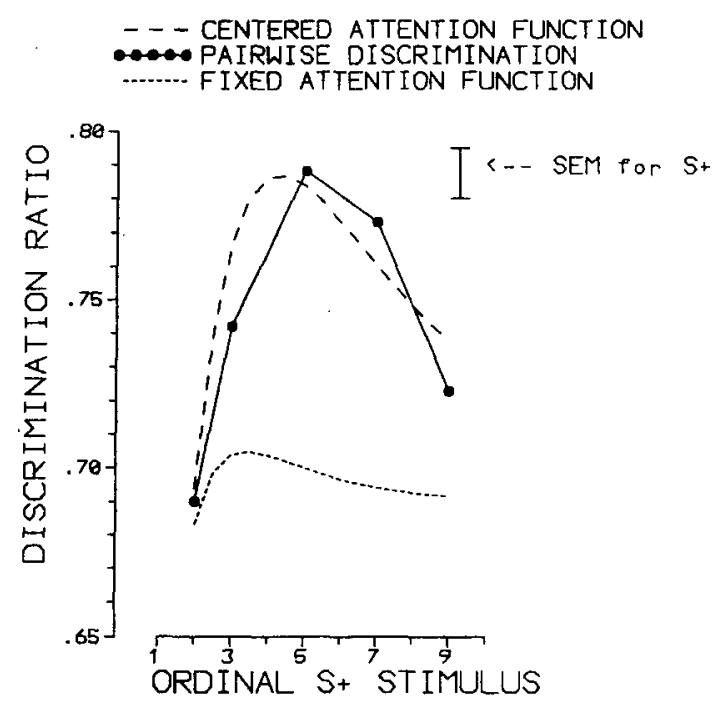

Figure 5. Group results from Experiment 2 and theoretical functions. Solid line: discrimination performance for each $\mathbf{S}+$ condition. Coarse dashed line: prediction for an attention function centered within the criterial range for each pair of $S-$ and $\mathbf{S}+$. Fine dashed line: Prediction for an attention function fixed between the closest $S-$ and $S+$. The vertical bar represents plus and minus one standard error of the mean for discrimination ratios. 
line is the prediction when the attention function is fixed between $\mathrm{S}-$ and the closest $\mathrm{S}+$ along the width continuum. There is obviously close agreement between the obtained results and the first prediction of an attention function whose peak moves to the dimensional border for each session. As was reported in Experiment 1, when the criterial range of $\mathrm{S}-$ and $\mathrm{S}+$ changes, the peak of the attention function moves to the new dimensional border.

In summary, Experiment 2 demonstrates that positive dimensional contrast can occur in simple discrimination settings involving small numbers of stimuli. Increased spacing of $\mathrm{S}-$ and $\mathrm{S}+$ appears to change the dispersal of attentional resources available for the task, even with minimal numbers of training stimuli. Moreover, the attentional model does a good job of accounting for the results of Experiment 2 on the basis of the simplest assumption about the centering of the peak of the attention function at the dimensional border.

\section{GENERAL DISCUSSION}

The attentional model of dimensional contrast has explicit spatial properties, based on the way in which stimulus similarities and dissimilarities are internally represented and on the application of a gradient of attentional resources to the internal representation. Both Equation 1 and Equation 2 are based on the separation, or distance between, stimuli along a physical dimension. It is these spatial properties that predict the occurrence of dimensional contrast (see Hinson \& Tennison, 1997).

Implications of the spatial properties of the attentional model were directly examined in the present experiments. According to the model, the distances between positive and negative stimuli within the internal representation determine the peak of the attention function. Experiments 1 and 2 provide clear evidence that the peak location of the attention function moves when the criterial range changes. In both of these experiments, the best fits of the model were obtained by assuming that the peak of the attention function moves toward the new value of $S+$. Thus, the gradient form of attentional allocation presumed by the model was essential to properly account for performance.

The attentional model also implies that the greater the range of variation of stimuli throughout the internal representation, the greater the dispersion of limited attentional resources. As a result, the relative level of attention in a given region should decline as stimulus range increases. In several previous studies, we have reported that overall performance declines as stimulus range increases (e.g., Hinson \& Higa, 1989b; Hinson \& Lockhead, 1986; Hinson \& Tennison, 1998; Tennison \& Hinson, 1993). The present experiments provide more evidence that such range effects are due to changes in the allocation of attentional resources. For example, Experiment 1 showed that a single attentional parameter of the model decreased as stimulus range increased, thereby producing the observed decline in performance. A similar attentional factor ac- counted for the results of Experiment 2, in which only one positive and one negative stimuli were used during each session.

A further implication of the attentional model is the putative tradeoff resulting from increased spatial separation of stimuli within the internal representation. Equation 1 predicts that discrimination should improve as $\mathrm{S}-$ and $\mathrm{S}+$ become more widely separated, whereas Equation 2 predicts that discrimination should decline as attentional resources are dispersed more widely. The predicted interaction between Equation 1 and Equation 2 was observed in both Experiment 1 and Experiment 2, where separation between negative and positive stimuli was manipulated.

Although Blough's incremental model of dimensional contrast is generally accurate (e.g., D. S. Blough, 1975), it does not predict the stimulus spacing effects found in the present experiments. The model anticipates an effect of criterial range (e.g., P. M. Blough, 1980), but not the interaction between criterial range and stimulus range. The present findings suggest that Blough's account may be omitting attentional processes that contribute to dimensional contrast. Perhaps it is these missing attentional processes that lead the incremental model to underestimate the obtained magnitude of dimensional contrast in many settings (see D. S. Blough, 1983).

It may appear that the attentional model has too many free parameters and can, therefore, easily accommodate any finding. We believe that this is not a fair interpretation. To begin with, the original model in Equation 3 has three free parameters. At a minimum, two parameters are necessary to account for a bitonic function, such as the positive dimensional contrast function. Moreover, the free parameter $x$ in Equation 1 is a scaling factor that should be constant for a given stimulus dimension (see, also, Hinson $\&$ Tennison, 1997). The results in Experiments 1 and 2 show that parameter $x$ in Equation 1 can be treated as a constant, once we have established its value in one setting. This leaves the two parameters of Equation 2 as free parameters.

The revised model in Equation 7 adds a parameter, to allow variation in the location of the peak of the attention function. To better constrain the fit, we held two parameters constant and accounted for all the results with only two free parameters in the revised model. Although it is still possible that this additional parameter allows too much flexibility in the model, we would argue to the contrary. Our evaluation of fit of the model yielded values of $c$ consistent with our interpretation of the attention function. That is, $c$ was approximately zero when criterial range was small, and $c$ increased well above zero when criterial range increased. If the new parameter were an arbitrary addition that simply allowed us to capture more variance in the data, there would be no reason to expect $c$ to have provided these consistent values. Instead, the value of $c$ could have as easily varied randomly for each data set, providing an improved fit, without a sensible theoretical interpretation. 
But we must admit shortcomings in our present treatment. Our model is a functional model that summarizes a set of presumptively attentional processes. Merely fitting results to this model does not allow us to specify the presumed attentional mechanisms responsible for performance. Moreover, in the present discussion, much of our argument for attentional processes is based on indirect evidence. We believe that future studies should employ more direct attentional manipulations. D. S. Blough and P. M. Blough (1997) provide a thorough summary of ways to explore the impact of attentional processes during visual discrimination tasks. Attentional manipulations, such as those involving priming or precuing techniques, can provide more decisive evidence that dimensional contrast and related phenomena are, in fact, due to attentional factors.

\section{REFERENCES}

BALSAM, P. D. (1988). Selection, representation, and equivalence of controlling stimuli. In R. C. Atkinson, R. J. Herrnstein, G. Lindzey, \& R. D. Luce (Eds.), Stevens' handbook of experimental psychology (Vol. 2, pp. 111-166). New York: Wiley.

Blough, D. S. (1975). Steady-state generalization and a quantitative model of operant generalization and discrimination. Journal of Experimental Psychology: Animal Behavior Processes, 104, 3-21.

BLougH, D. S. (1983). Alternative accounts of dimensional stimulus control. In M. Commons, R. Herrnstein, \& A. Wagner (Eds.), Quantitative analyses of behavior (Vol. 4, pp. 59-72). Cambridge, MA: Ballinger.

Blough, D. S., \& Blough, P. M. (1997). Form perception and attention in pigeons. Animal Learning \& Behavior, 25, 1-20.

Blough, P. M. (1980). Behavioral and dimensional contrast in rats. Journal of the Experimental Analysis of Behavior, 33, 345-357.

BoneAu, C. A., \& CoLe, J. L. (1967). Decision theory, the pigeon, and the psychophysical function. Psychological Review, 74, 123-135.

Briand, K. A., \& Klein, R. M. (1987). Is Posner's 'beam' the same as Treisman's "glue": On the relation between visual orienting and feature integration theory. Journal of Experimental Psychology: Human Perception \& Performance, 13, 228-241.

Catania, A. C., \& Gill, C. A. (1964). Inhibition and behavioral contrast. Psychonomic Science, 1, 257-258.

Essock, S. M., \& BLovGH, D. S. (1977). Effects of stimulus spacing on steady state gradients of inhibitory stimulus control. Animal Learning \& Behavior, 5, 174-176.

FARTHING, G. W. (1974). Behavioral contrast with multiple positive and negative stimuli on a continuum. Journal of the Experimental Analysis of Behavior, 22, 419-425.

Hays, W. L. (1988). Statistics (4th Ed.). New York: Holt, Rinehart \& Winston.

HeinemanN, E. G., \& Avin, E. (1973). On the development of stimulus control. Journal of the Experimental Analysis of Behavior, 20, 183 . 195.

Heinemann, E. G., Avin, E., Sullivan, M. A., \& Chase, S. (1969). Analysis of stimulus generalization with a psychophysical method. Journal of Experimental Psychology, 80, 215-224.

Heinemann, E. G., \& Chase, S. (1975). Stimulus generalization. In W. K. Estes (Ed.), Handbook of learning and cognitive processes (Vol. 2, pp. 305-349). Hillsdale, NJ: Erlbaum.
Heinemann, E. G.. \& Chase, S. (1990). A quantitative model for pattern recognition, In M. L. Commons, R. J. Herrnstein, S. M. Kosslyn, \& D. B. Mumford (Eds.), Quantitative analyses of behavior (Vol. 9, pp. 109-126). Hillsdale, NJ: Erlbaum.

Hinson, J. M. (1988). Absolute and relative measures of dimensional contrast. Journal of the Experimental Analysis of Behavior, 50, 249260.

Hinson, J. M., Cannon, C. B., \& Tennison, L. R. (1999). Orthogonal stimulus variation and attention in dimension contrast. Animal Learning \& Behavior, 27, 181-189.

Hinson, J. M., \& HigA, J. J. (1989a). Discrete and continuous measures of dimensional stimulus control. Journal of the Experimental Analysis of Behavior, 51, 199-214.

Hinson, J. M., \& HiGA, J. J. (1989b). Stimulus variation and dimensional contrast. Animal Learning \& Behavior, 17, $31-38$.

HinSON, J. M., \& LockHEAD, G. R. (1986). Range effects in successive discrimination. Journal of Experimental Psychology: Animal Behavior Processes, 12, 270-276.

Hinson, J. M., \& Malone, J. C., JR. (1980). Local contrast and maintained generalization. Journal of the Experimental Analysis of Behavior, 34, 263-272.

Hinson, J. M., \& Tennison, L. R. (1997). An attentional model of dimensional contrast. Journal of Experimental Psychologv: Animal Behavior Processes, 23, 295-311.

Hinson, J. M., \& TEnnison, L. R. (1998). Range effects using instru mental choice procedures. Animal Learning \& Behavior, 26, 60-75.

HiRST, W., \& KaLmar, D. (1987). Characterizing attentional resources. Journal of Experimental Psychology: General, 116, 68-81.

LABERGE, D., \& BROWN, V. (1989). Theory of attentional operations in shape identification. Psychological Review, 96, 101-124.

Navon, D. (1984). Resources-A theoretical soup stone? Psychological Review, 91, 216-234.

PEARCE, J. M., \& Redhead, E. S. (1993). The influence of an irrelevant stimulus on two discriminations. Journal of Experimental Psychology: Animal Behavior Processes, 19, 180-190.

Posner, M. I., SNyder, C. R. R., \& Davidson, B. J. (1980). Attention and the detection of signals. Journal of Experimental Psychology: General, 109, 160-174.

Rescorla, R. A., \& Wagner. A. R. (1972). A theory of Pavlovian conditioning: Variations in the effectiveness of reinforcement and nonreinforcement. In A. H. Black \& W. F. Prokasy (Eds.), Classical conditioning II: Current research and theory (pp. 64-99). New York: Appleton-Century-Crofts.

REYNOLDS, G. S. (1961). Contrast, generalization, and the process of discrimination. Journal of the Experimental Analysis of Behavior, 4 , 289-294.

SHEPARD, R. N. (1987). Toward a universal law of generalization for psychological science. Science, 237, 1317-1323.

SPENCE, K. W. (1937). The differential response in animals to stimuli varying within a single dimension. Psychological Review, 44, 430444.

SYSTAT: THE SYSTEM FOR STATISTICS [Computer software]. (1990). Evanston, IL: SYSTAT.

TENNison, L. R., \& Hinson, J. M. (1993). Range effects in transient and maintained generalization gradients. Behavioural Processes, 29, 201 216.
(Manuscript received September 17, 1998; revision accepted for publication May 3, 1999.) 